\title{
Age and sensory processing abnormalities predict declines in encoding and recall of temporally manipulated speech in high-functioning adults with ASD.
}

\author{
Jennifer L. Mayer ${ }^{1} \&$ Pamela F. Heaton ${ }^{2}$
}

\begin{abstract}
While temporal and perceptual processing abnormalities, identified in a number of electrophysiological and brain imaging studies of individuals with (ASD), are likely to impact on speech perception, surprisingly little is known about the behavioral outcomes of such abnormalities. It has been hypothesized that rapid temporal processing deficits may be linked to impaired language development through interference with acoustic information during speech perception. The present study aimed to investigate the impact of temporal changes on encoding and recall of speech, and the associated cognitive, clinical, and behavioral correlates in adults with ASD. Research carried out with typically developing (TD) adults has shown that word recall diminishes as the speed of speech increases, and it was predicted that the magnitude of this effect would be far greater in those with ASD because of a preexisting rapid temporal processing deficit. Nineteen high-functioning adults with ASD, and age- and intelligence-matched TD controls performed verbatim recall of temporally manipulated sentences. Reduced levels of word recall in response to increases in presentation speed were observed, and this effect was greater in the older participants in the ASD group than in the control group. This is the first study to show that both sensory abnormalities and aging impact on speech encoding in ASD. Auditory processing deficits in ASD may be indicative of an association with the sensory abnormalities and social and communication impairments characterizing the disorder.
\end{abstract}

Keywords: Autism Spectrum Disorders; Speech Perception; Auditory Processing; Aging

${ }^{1}$ University of Roehampton, Department of Psychology, Whitelands College, Holyborne Avenue, London, SW15 4JD, UK. ${ }^{2}$ Goldsmiths College, Department of Psychology, 8 Lewisham Way, New Cross, London SE14 6NW, UK.

CORRESPONDING AUTHOR: Dr Jennifer Mayer, email address: Jennifer.mayer@roehampton.ac.uk, phone: +44 (0)20 83923661

* This is a preprint of an article published in: Mayer, J., \& Heaton, P. (2013). Age and Sensory Processing Abnormalities Predict Declines in Encoding and Recall of Temporally Manipulated Speech in High-Functioning Adults with ASD. Autism Research, 1-10. doi:10.1002/aur.1333 http://onlinelibrary.wiley.com/doi/10.1002/aur.1333/abstract 


\section{INTRODUCTION:}

Autism Spectrum Disorders (ASD) are neurodevelopmental disorders characterized by impairments in social interaction, deficits in cognitive flexibility, and deficits in communication and language abilities. The Diagnostic and Statistical Manual of Mental Disorders (DSM-IV) further defines the communication cluster in ASD specifying that a significant delay in language or a clear deficit in the ability to carry on a conversation with others be present to elicit an ASD diagnosis (American Psychiatric Association, 2000). Whilst researchers have estimated that between 25$50 \%$ of individuals with ASD never acquire functional language (Gillberg \& Coleman, 2000; Klinger, et al., 2002), the presentation of language impairments within this population is extremely diverse and although intellectually able adults with ASD may experience difficulties carrying on a conversation, their scores on formal tests of verbal intelligence may well be in the normal range or higher.

Whilst temporal and perceptual processing abnormalities, identified in a number of electrophysiological and brain imaging studies of individuals with ASD (Fujikawa- Brooks et al., 2010; Gervais et al., 2004; Källstrand et al., 2010; Lepistö et al., 2009; Whitehouse \&Bishop, 2008), are likely to impact on speech perception surprisingly little is known about the behavioural outcomes of such abnormalities. However, the importance of addressing this question is highlighted by research showing that language processing difficulties limit the psychosocial and vocational opportunities of intellectually able adults with ASD (Howlin, et al., 2005). Research aiming to elucidate inter-relationships between information processing abnormalities and core communication deficits in individuals with ASD will make an important contribution to the theoretical and empirical base that informs the development of intervention services for these individuals.

Temporal aspects of auditory stimuli carry important information. For example, Rosen (1992) proposed that temporal cues are the primary component upon which speech perception is based and studies have demonstrated that deficiencies in speech perception are often associated with deficits discriminating temporal auditory features (Kujala et al., 2000). Furthermore, changes to the temporal parameters of speech affect other perceptual components of the speech signal; increasing the rate of natural speech also involves changes at the syllable, word, and sentence levels that disrupt the relationship between their timing and that of speech units (Janse, 2004). Several studies have examined temporal perception of speech through rapid speed processing in typically developing individuals and have observed significant declines in rates of performance with increasing speech rate (Stine, et al., 1986; Tun, et al., 1992; Tun, 1998; Wingfield, 1975; Wingfield, et al., 1985).

Impairments in temporal processing are often noted in individuals with ASD. A recent study by Kwakye and colleagues (2011) using temporal order judgment tasks provided evidence for impairments in both multisensory and auditory temporal processing in children with ASD. Their findings are consistent with other behavioural studies and electrophysiological findings of reduced mismatch negativity in response to duration changes in non-speech sounds (Lepistö et al., 2005, 2006). Taken together, these results indicate atypical responses to temporal aspects of auditory information in ASD. Kwakye et al. (2011) suggested that these impairments could be due in part to an extended temporal processing window in individuals with ASD, which affects the rapid processing of sensory information.

Gepner \& Féron (2009) put forth a tempo-spatial processing hypothesis to explain various degrees of disability in ASD. Within the auditory domain, the authors noted evidence of impairments in speech flow perception and segmentation in 
children with ASD (Gepner \& Massion, 2002) and increased phoneme categorization performance when phonemes were produced at reduced speeds (Tardif et al., 2002). Their hypothesis suggests that rapid changes in the environment, acting on one or more sensory modalities, are implicated in processing impairments in children and adolescents with ASD. However, the extent to which this hypothesis generalises to adults with ASD has not been investigated. Rapid processing impairments in the auditory domain may well result in difficulties with verbal comprehension and impairments in language abilities (OramCardy, et al., 2005). This is particularly concerning as the ability to integrate temporal information is hypothesised to be vital in the development of those social functions that are impaired in individuals with ASD (Gepner \& Tardif, 2006).

Speech processing involves the rapid decoding of a constantly changing signal that must occur in real time. Thus, it is not surprising that individuals who experience temporal processing difficulties overall would have more difficulty with rapid speech. Previous research (Stine et al, 1986; Tun et al., 1992; Tun, 1998; Wingfield et al., 1985) found that elderly adults demonstrated steeper declines in rates of performance with increasing speech rate in comparison to younger individuals. Speech rate is normally under the control of the speaker rather than the listener, and impairments in rapid speech processing could therefore have a direct impact on one's social communication abilities. Studies by Laine et al. (2008 \&2009) attempted to alleviate the effects of rapid processing impairments in children with ASD by slowing the auditory presentation of sentences. Verbal comprehension was enhanced during slow speech rates, especially in children with low-functioning autism. Thus, temporal manipulations to speech through increasing the rate of presentation may well uncover increased speech and information processing abnormalities in adults with ASD.

One reason we might expect temporal speech manipulations to result in increased processing abnormalities in adults with ASD is the presence of sensory atypicalities, which are known to be pervasive and continue into adulthood (Leekam, et al., 2007; Crane, et al., 2009). Based on Gepner \& Féron's (2009) hypothesis and Kwakye et al.'s (2011) findings it is plausible that increased sensitivity to and awareness of sensory information may make it more difficult for adults with ASD to process rapid speech changes. The recent line of research into aging in ASD also raises interesting questions giventhe previously cited findings regarding aging and rapid speech processing in typically developing individuals. Neurologial studies have highlighted correlations between atypical cortical thickness and age in individuals with ASD that are not present in typically developing controls (Wallace, et al., 2010; Doyle-Thomas \& Duerden, 2013). However, little research exists regarding the possible behavioural effects of age-related neurological atypicalities in adults with ASD.

The following experiment aims to investigate the impact of temporal changes on encoding and recall of speech in ASD and typically developing adults. Research carried out with typically developing adults has shown that word recall diminishes as the speed of speech increases and it is predicted that the magnitude of this effect will be far greater in those with ASD due to a pre-existing rapid temporal processing deficit. In addition to analysing accuracy scores, the present study also incorporates the use of recall times in order to examine some of the more subtle processing differences. The present study also aims to explore the relationship between cognitive, behavioural, and clinical correlates (i.e. sensory processing abnormalities, autistic traits, chronological age, etc.) and memory and recall for rapidly presented sentences using regression analyses. 


\section{METHODS}

\section{Participants}

19 adults (4 female/15 male) with high-functioning ASD (with IQ scores of 70 or above) were recruited to the study. Their chronological ages ranged between 23 years 9 months and 59 years 8 months. Whilst the oldest adult in the ASD group was seven years older than the oldest adult in the TD group, he was not an outlier on any of the background assessments or experimental measures. As the results did not change when his data were removed, they were retained in the analyses. All of the adults in the ASD group were recruited from local support groups or had previously participated in research at Goldsmiths College, London, UK. All ASD participants' pre-existing diagnoses were confirmed by the first author using ADOS module 4 . 19 adults with typical development were matched to the ASD group on age, gender, receptive vocabulary, working memory, as well as on verbal, performance, and full scale IQ scores (see following section). Their chronological ages ranged between 25 years 1 month and 52 years 8 months. All control participants' were screened for ASD using the Autism Spectrum Quotient (Baron-Cohen, et al., 2001). Scores on this test ranged from 3 to 21, which is well below the cut-off score of 32 proposed by BaronCohen et al. (2001). See Table 1 for the background data summary for the two groups.

\section{Cognitive Correlates}

Weschler Abbreviated Scales of Intelligence

The Weschler Abbreviated Scales of Intelligence (WASI) (Wechsler, 1999) was used as a measure of intellectual and cognitive functioning.

\section{Peabody Picture Vocabulary}

The Peabody Picture Vocabulary Test (PPVT) (Dunn \& Dunn, 1997) was used as a measure receptive vocabulary.

\section{Working Memory}

The ability to encode information into working memory plays an important role in the speed and accuracy with which one can rapidly process information (Stine, et al., 1986; Tun, 1998). In order to assess participants' working memory capacity, the backwards digit span subtest from the Weschler Adult Intelligence Scale - Fourth Edition (WAIS-IV) (Wechsler, 2008) was used.

\section{Behavioural Correlates}

\section{Communication Checklist - Self Report}

The Communication Checklist - Self Report (CC-SR) (Bishop, et al., 2009) was administered to provide information on any difficulties in speech, language, or interaction that may affect the participants' communication abilities. The CC-SR is a 70-item self-report questionnaire, which examines three factors of communication: Language Structure ("I make false starts or search for the right word"), Pragmatic Skills ("I am told that I keep talking about things that others are not interested in"), and Social Engagement ("I find it hard to know when people are upset or annoyed"). Higher scores on the CC-SR indicate an increased level of communication difficulties. The ASD group scored higher than the TD group on all of the CC-SR measures, demonstrating a significantly greater level of self-reported communication difficulties. 


\section{Adult/Adolescent Sensory Profile}

Whilst sensory abnormalities are not currently included in DSM-IV-TR (American Psychiatric Association, 2000) they are widely prevalent ASD (e.g. Leekam, et al., 2007) and may be implicated in language processing difficulties in ASD. Therefore measures of sensory abnormalities using the Adult/Adolescent Sensory Profile (SP) test (Brown \& Dunn, 2002) were also obtained. The SP is a 60 item self-report questionnaire that examines sensory processing patterns across six sensory processing categories including: taste/smell, movement, visual, touch, activity, and auditory processing. Participants' raw scores across the 6 categories are used to derive their quadrant scores identified as: Low Registration ("I don't get jokes as quickly as others"), Sensation Seeking ("I like to wear colourful clothing"), Sensory Sensitivity ("I am distracted if there is a lot of noise around"), and Sensation Avoiding ("I stay away from crowds"). Higher scores within each quadrant represent increased sensory abnormalities. The ASD group had higher scores than the TD group on three of the four quadrants, indicating a greater level of sensory abnormalities.

\section{Clinical Correlates}

\section{Adult Autism Spectrum Quotient}

In order to assess the self-reported levels of autistic traits in participants the Adult Autism Spectrum Quotient (AQ) (Baron-Cohen et al., 2001) was administered. The AQ is a 50 item questionnaire which examines 5 factors: Social Skills ("I would rather go to a library than a party"), Attention Switching ("I frequently get so absorbed in one thing that I lose sight of other things"), Attention to Detail ("I often notice small sounds when others do not"), Communication ("Other people frequently tell me that what I've said is impolite, even though I think it is polite"), and Imagination ("When I'm reading a story, I find it difficult to work out the characters' intentions"). Within the AQ autistic-like behaviour is characterised by poor social, communication, or imagination skills, exceptional attention to detail, and either poor attention switching or a strong focus of attention (Baron-Cohen et al., 2001). The ASD group scored higher than the TD group on all of the AQ measures, which demonstrates a significantly greater level of self-reported autistic traits in the participants with ASD.

\section{Autism Diagnostic Observation Schedule}

All of the ASD individuals who participated in the present study had previously been diagnosed by clinicians in accordance with Diagnostic and Statistical Manual of Mental Disorders (4 ${ }^{\text {th }}$ ed., rev.; American Psychiatric Association, 2000). ASD participants' pre-existing diagnoses were confirmed by administering the Autism Diagnostic Observation Schedule (ADOS) module 4(Lord, et al., 2001). The ADOS provides a score representing autistic symptom severity in the areas of: Communication, Reciprocal Social Interaction, Imagination and Creativity, and Repetitive Behaviours. Of the 19 ASD participants recruited, two did not meet overall diagnostic criteria on the ADOS. However, as all participants had previously been diagnosed by a clinician and the results from the background assessments and the experimental task did not change if those individuals were excluded, they were retained in the final sample.

INSERT TABLE 1 ABOUT HERE 


\section{Experimental Methods}

\section{Experimental Stimuli}

Sentence stimuli consisted of 30, 15-word sentences randomly selected from the 60 sentences used by Tun et al. (1992) (e.g. "The setting of Greece and its ancient monuments make it a fascinating place to visit"). The sentences were recorded by an adult British English speaking female and manipulated using PRAAT (Boersma, 2001) to generate three different speed conditions: normal speech (140 words per minute (wpm)), moderate speed (200 wpm) and fast speed $(280 \mathrm{wpm})$. Normal speech acted as the baseline condition and was only manipulated by adjusting the original sentences to the mean intensity (perceived volume) and a median pitch of $200 \mathrm{~Hz}$. The moderate and fast speed conditions were generated using electronic time compression to reduce the normal speech sentences to $70 \%$ and $50 \%$ of their original length.

\section{Procedure}

Participants were administered 3 practice sentences, one under each condition, and asked to perform a verbatim recall immediately following the end of the recorded sentence. Participants were instructed to repeat as much of the sentence as they could in the order that they had heard it. 30 experimental sentences followed in the same format. During the experimental trials, participants' responses were timed and recorded for later analysis. Participants received one point for each correct word. No points were awarded for words that were either incorrect or in the wrong order. Raw scores for each condition were converted to percentages for the analysis.

Analysis

Discrepancy scores were generated for each participant in order to account for any individual differences in working memory, language comprehension, or speech rate that may have affected their performance. Participants' percentage correct scores on the perceptual manipulation conditions were subtracted from their scores on the normal speech (baseline) condition in order to calculate their individual levels of change in response to speed manipulations.

A factorial analysis of variance (ANOVA) was used to analyse the data with the within-subjects factor of speed condition (2 levels; moderate speed and fast speed) and between-subjects factor of group (2 levels; ASD and TD). The dependent variable was the discrepancy scores for each participant across the 10 sentences in each speed condition. Further correlation and regression analyses were conducted to explore the relationship between cognitive, behavioural, and clinical correlates and memory and recall for rapidly presented sentences. Due to the exploratory nature of the regression analyses and the lack of previous literature on which to base a priori predictions a backwards stepwise entry method was employed.

\section{RESULTS}

\section{Accuracy and Recall Time Analyses}

Means, standard deviations, and ranges for the accuracy percentage correct scores and discrepancy scores as well as recall time raw scores and discrepancy scores across speed manipulations are shown in table 2 .

INSERT TABLE 2 ABOUT HERE 
There was a highly significant main effect of speed manipulation on participants' accuracy during sentence recall, $F(1,38)=9.29, p<0.01$.Participants' performance indicated a significantly higher level of difficulty when encoding and recalling speech spoken at a fast rate of speed in comparison to moderate speed $(\mathrm{M}=-$ $0.47, \mathrm{SD}=8.27$ for moderate; $\mathrm{M}=3.44, \mathrm{SD}=8.32$ for fast speech). However, there was no significant main effect of speed manipulation on participants' sentence recall speed, $F(1,38)=1.08, p=0.305(\mathrm{M}=4.14, \mathrm{SD}=28.01$ for moderate; $\mathrm{M}=6.49, \mathrm{SD}=23.08$ for fast speed).

In order to examine whether participants experienced significantly more difficulty in the two conditions with speed manipulations than normal speech alone, a one-sample t-tests were conducted. A mean value of 0 , which would indicate identical performance when recalling perceptually manipulated speech and normal speech, was used. Accuracy and reaction time results revealed a significant difference between discrepancy scores on the fast, $(t(37)=2.55, p<0.05$ for accuracy; $t(37)=3.12, p<0.01$ for recall time) speed condition and 0 , but not on the moderate, $(t(37)=-0.35, p=0.726$ for accuracy; $t(37)=1.59, \mathrm{p}=0.121$ for recall time) speed condition and 0 . Thus, the results showed that individuals experienced significantly more difficulty with both accuracy and recall time during the fast condition in comparison to normal speech, but were equally able to encode and recall moderately fast and normal speeds of speech.

Although ASD individuals experienced slightly more difficulty with accuracy when encoding and recalling sentences with speed manipulations in comparison to typically developing participants $(\mathrm{M}=2.82, \mathrm{SD}=7.62$ for $\mathrm{ASD}$, and $\mathrm{M}=0.14, \mathrm{SD}=6.93$ for TD) this was not statistically significant $F(1,38)=1.29, p=0.264$, and there was no significant speed manipulation by group interaction, $F(1,38)=0.12, p=0.735$. There was also no significant main effect of group on the participants' sentence recall speed, $F(1,38)=0.01, p=0.925$. However, results indicated that both groups were recalling the sentences faster during the speed manipulation conditions in comparison to their baseline recall speeds. $(M=5.12, S D=14.13$ for $A S D$, and $M=5.50, S D=11.07$ for $T D)$.

There was however, a significant speed manipulation by group interaction in the recall time analysis, $F(1,38)=5.25, p<0.05$ (Fig. 1). In order to further examine the interaction two post-hoc t-tests were conducted. Results revealed a non-significant trend towards ASD participants recalling sentences more slowly during the moderate speed condition in comparison to the fast speech condition, $t(18)=-2.00$, $p=0.060$.Typically developing participants, on the other hand, tended to recall sentences slower during the fast speech condition, although not significantly so, $t(18)=1.12, p=0.274$. The second group of post-hoc t-tests examined performance within each condition, across groups. No significant differences were found $(t(18)=1.159, p=0.254$ for fast speed; $t(18)=-1.073, p=0.290$ for moderate speed).

INSERT FIGURE 1 ABOUT HERE

\section{Regression Analyses}

Whilst difficulties encoding and recalling temporally manipulated speech were not observed at the group level in the ASD sample, scores for this group did appear to be more variable. As an important aim of the study was to explore the extent that variations in performance on the speed manipulation paradigm were associated with specific clinical, cognitive and behavioural factors (described in the methods section), correlation analyses were first carried out on the data. Both groups experienced more difficulty encoding and recalling fast but not moderate speech therefore the correlation and regression analyses focused only on the fast speech discrepancy 
scores. Significant correlations are shown in table 3. All of the variables that were significantly correlated with performance on the experimental task were used in regression analyses.

INSERT TABLE 3 ABOUT HERE

A multiple linear regression was performed to examine the extent to which the significantly correlated cognitive, behavioural, and clinical factors shown in table 3 explained the variance in encoding and recall of fast speech in the ASD group. The results revealed a significant linear relationship between ASD participants' accuracy discrepancy scores during the fast speed condition and the predictor variables with a multiple correlation of $0.96,\left[\mathrm{~F}(1,19)=34.12, p<0.001\right.$; adjusted $\left.\mathrm{R}^{2}=0.886\right]$ (table 4). Thus, $88 \%$ of the variability in ASD participants' accuracy discrepancy scores during the fast speed condition was predicted by their chronological age and scores on the sensory sensitivity subscale of the Sensory Profile. Higher chronological age and levels of sensory sensitivity predicted an increase in an ASD individual's discrepancy scores indicating increased perceptual disturbance when encoding and recalling speech spoken at a fast speed.

INSERT TABLE 4 ABOUT HERE

\section{DISCUSSION}

One of the primary aims of the present study was to increase our understanding of the effect of temporal manipulations on speech encoding and recall in individuals with ASD. Although no overall group differences emerged within either the accuracy or recall time analyses, this is not necessarily surprising given that both groups possessed higher than average levels of intelligence and good verbal skills. This result does not appear to reflect insensitivity in the paradigm as there were significant differences between accuracy but not recall time discrepancy scores on the moderate and fast speed manipulations when the analysis was carried out on the data from the whole sample. Thus, individuals within both groups recalled significantly fewer correct words when the speed of speech was twice as fast as normal, but not when sentences were produced at a moderately fast speed. Furthermore, accuracy results indicated that individuals were experiencing significant levels of perceptual disturbance from the fast temporal manipulation in comparison to normal speech. Thus individuals across both groups experienced more difficulty encoding and recalling sentences when the speed of speech increased. Recall time analyses also revealed a significant interaction between temporal manipulations and group. Trends suggested that ASD participants recalled sentences slower during the moderate speech condition in comparison to the fast speech condition, whilst typically developing individuals tended to recall sentences slower during the fast speech condition. It is unclear whether this result is a consequence of different auditory processing strategies in the two groups or indicative of subtle temporal processing abnormalities in individuals with ASD. Future studies utilising electrophysiological methodologies may be able to address this question.

Another aim of the present study was to explore the cognitive, behavioural, and clinical correlates associated with auditory temporal processing in typically developing and ASD individuals. Individuals with ASD and typical controls demonstrated very different profiles on the regressions and correlations carried out on the experimental and background measures. First, regressions revealed a significant 
positive relationship between age and increased perceptual disturbance from fast speech in the ASD group but not the control group, despite the fact that the two groups were matched on mean age and range. Although previous research (Wingfield et al. 1985; Stine et al., 1986; Tun et al., 1992; Tun, 1998) found that elderly typical adults demonstrated steeper declines in rates of performance with increasing speech rate in comparison to younger individuals, their elderly cohort extended far beyond the age range of the participants tested in the present study. It is therefore unsurprising that the typically developing participants who completed the present experiment did not show the declines reported in these studies. The results showing an age related decline in the ASD group may suggest that individuals with this disorder are more susceptible to age related processing effects, such as cognitive slowing, than typically developing individuals. Furthermore, it is possible that such age-related effects are occurring at a younger age in adults with ASD compared to typical development. In light of Wallace et al.'s (2010) and Doyle-Thomas et al.'s (2013) findings of a relationship between abnormal cortical thickness and age in individuals with ASD, the present study also provides evidence to suggest there may be behavioural effects of atypical neurological aging in this disorder.

Correlations also showed that individuals with ASD who reported higher levels of difficulty across all subscales of the communication checklist also experienced significantly higher levels of perceptual disturbance from speech spoken at fast speed. Whilst a wealth of empirical data confirm that information processing abnormalities are a frequent, if not universal feature in ASD, they are not included in DSM-IV and are rarely considered in the context of core social and communication deficits ASD. The results from the current study indicate that there is a relationship between language and temporal processing abnormalities in individuals with ASD that extends to those on the very high-functioning end of the spectrum. A similar relationship was also found between sensory processing abnormalities, autistic traits relating to social skills and imagination, and accurate recall of sentences spoken at a fast speed. This finding was further supported by the regression analyses, which indicated that higher levels of self-reported sensory sensitivity in individuals with ASD predicted a significant increase in perceptual disturbance when encoding and recalling fast speech. These results suggest that auditory processing deficits in ASD may not just be a function of language impairment, but rather are indicative of an association with the sensory abnormalities and social and communication impairments characterising the disorder.

The relationship between performance on the experimental task and performance IQ suggests that the absence of clear group differences in the present study may very well be due to fact that only high-functioning individuals were included in the study. The results from the study suggested that whilst sensory/temporal processing difficulties did not impact on speech encoding and memory in ASD during early to mid-adulthood the decline in this skill, observed in typical development, occurs at significantly earlier periods in ASD. Future research should consolidate and extend this finding in studies of high-function children and older adults with ASD. Questions about the extent that speech encoding and memory is disrupted in lower-functioning individuals with ASD also merit investigation.

One of the primary aims of the present study was to increase understanding of heterogeneity in language skills in high-functioning adults with ASD by identifying the cognitive, clinical and behavioural correlates of deficits in speech perception. In order to address this question a number of background measures were utilized and on many of these variables (most notably the CC-SR and some of the subscales from the 
AQ) there was a much larger variance in the scores from the ASD group than the TD group. This is unsurprising given that a more heterogeneous profile, in terms of communication and autistic traits, would be expected within the ASD population. However, it is possible this factor contributed to the absence of any significant correlations between performance on the experimental task and the background measures in the TD group. Therefore the small correlations observed should be interpreted with caution and replication, especially focusing on sensory processing abnormalities and age should be sought.

Another limitation of the present study is the linguistic matching criteria of the sentence stimuli. The specific stimuli was selected because it had previously been used by Tun et al. (1992) to examine the age effects of rapid speech processing in TD individuals and thus provided the best opportunity for replication. Whilst the stimuli used by Tun et al. were matched for numbers of words and provided a high cognitive load, other linguistic factors that can affect speech encoding and recall such as number of syllables and word frequency were not controlled. However, the present study randomised the sentences in each speech condition across participants in order to account for any variability within the individual sentences. Future research should attempt to take a more rigorous linguistic approach by taking additional matching factors into account.

Whilst it may be argued that temporal manipulations of sentence stimuli are artificial and the results for the current study may not generalise to real life situations, research has indicated that natural fast speech is actually more difficult to process than artificially time-compressed speech. As Janse (2004) suggests, naturally fast speech is characterised by more general prosodic change and segmental overlap than slower speech. It is therefore plausible to suggest that high-functioning individuals with ASD may experience more difficulties processing fast speech in their everyday lives, than the current results suggest. Our results showed that speech processing deficits are most marked in older individuals and those with increased levels of self reported sensory disturbance. Given the likely negative impact of such difficulties on the vocational and psychosocial opportunities of affected individuals, further study into temporal speech processing throughout the lifespan in high-functioning individuals with ASD is clearly merited.

\section{ACKNOWLEDGEMENTS:}

This research was carried out with the support of the Baily Thomas Trust. We are very grateful to all the ASD and typically developing adults who participated in our study. We would also like to thank Mr. Ian Hannett for his assistance creating the stimuli. 


\section{REFERENCES:}

American Psychiatric Association. (2000). Diagnostic and statistical manual of mental disorders (4th ed., t.). Washington, DC: American Psychiatric Association.

Baron-Cohen, S., Wheelwright, S., Skinner, R., Martin, J., \& Clubley, E. (2001). The Autism-Spectrum Quotient (AQ): Evidence from Asperger Syndrome/HighFunctioning Autism, Males and Females, Scientists and Mathematicians. Journal of Autism and Developmental Disorders, 31(1), 5-17. Springer.

Bishop, D. V. M., Whitehouse, A. J. O., \& Sharp, M. (2009). Communication Checklist - Self-Report. San Antonio, TX: The Psychological Corporation.

Boersma, P. (2001). PRAAT, a systems for doing phonetics by computer. Glot International.

Brown, C., \& Dunn, W. (2002). Adult/Adolescent Sensory Profile. San Antonio, TX: The Psychological Corporation.

Crane, L., Goddard, L., \& Pring, L. (2009). Sensory processing in adults with autism spectrum disorders. Autism: the international journal of research and practice, 13, 215-228. doi:10.1177/1362361309103794.

Doyle-Thomas, K., \& Duerden, E. (2013). Effects of age and symptomatology on cortical thickness in autism spectrum disorders. Research in Autism Spectrum Disorders, 7, 141-150.

Dunn, L. M., \& Dunn, M. (1997). Peabody Picture Vocabulary Test. Circle Pines, MN: American Guidance Service.

Fujikawa-Brooks S, Isenberg AL, Osann K, Spence MA, Gage NM. The effect of rate stress on the auditory brainstem response in autism: A preliminary report. International Journal of Audiology.2010;49(2):129-140. doi: $10.3109 / 14992020903289790$.

Gepner, B., \& Féron, F. (2009). Autism: a world changing too fast for a mis-wired brain? Neuroscience and biobehavioral reviews, 33(8), 1227-42. doi:10.1016/j.neubiorev.2009.06.006

Gepner B. \& Massion, J. (dir by), 2002. L'autisme :unepathologie du codagetemporel ? Trav. Interdisciplin. Lab Parole Lang. 21, 177-218. IN: Gepner, B., \&Féron, F. (2009). Autism: a world changing too fast for a mis-wired brain? Neuroscience and biobehavioral reviews, 33(8), 1227-42.

doi:10.1016/j.neubiorev.2009.06.006

Gepner, B. \& Tardif, C., 2006.Autism, movement, time and thought. E-motion missight and other temporo-spatial processing disorders in autism. In: Vanchevsky, M. (Ed.), Frontiers in Cognitive Psychology. Nova Science Publishers, New York, pp. 1-30.

Gervais, H., Belin, P., Boddaert, N., Leboyer, M., Coez, A., Sfaello, I., Barthélémy, C., et al. (2004).Abnormal cortical voice processing in autism. Nature, 7(8), 801-803. doi:10.1038/nnl291

Gillberg, C., \& Coleman, M. (2000). The Biology of the Autistic Syndromes. John Wiley \& Sons.

Howlin, P., Alcock, J., \& Burkin, C. (2005). An 8 year follow-up of a specialist supported employment service for high-ability adults with autism or Asperger syndrome. Autism, 9 (5), 533-549.doi:10.1177/1362361305057871

Janse, E. (2004). Word perception in fast speech: artificially time-compressed vs. naturally produced fast speech. Speech Communication, 42(2), 155 173.doi:10.1016/j.specom.2003.07.001 
Källstrand, J., Olsson, O., Nehlstedt, S.F., Skold, M.L., Nielzen, S., 2010. Abnormal audi- tory forward masking pattern in the brainstem response of individuals with Asperger syndrome. Neuropsychiatry Disorders and Treatment 6, 289-296.

Klinger, L., Dawson, G., \& Renner, P. (2002). Autistic disorder. In E. Mash and R. Barkley (Eds). Child Psychopathology. (pp 409-454). New York: Guilford Press.

Kujala, T., Myllyviita, K., Tervaniemi, M., Alho, K., Kallio, J., \& Näätänen, R. (2000). Basic auditory dysfunction in dyslexia as demonstrated by brain activity measurements. Psychophysiology, 37(2), 262-266. Blackwell Publishing.

Kwakye, L. D., Foss-Feig, J. H., Cascio, C. J., Stone, W. L., \& Wallace, M. T. (2011). Altered auditory and multisensory temporal processing in autism spectrum disorders. Frontiers in integrative neuroscience, 4(January), 129. doi:10.3389/fnint.2010.00129

Laine', F., Tardif, C., Gepner, B., 2008.Slowing Down Biological Motion and Speech Sounds Enhances Imitation and Verbal Comprehension in Children and Adolescents with Autism. Poster at the Autism Neuroscience Conference, Royal Society, London, October.

Laine', F., Rauzy, S., Gepner, B., Tardif, C., 2009. Prise en compte des difficulte' sdetraitement des informationsvisuellesetauditivesrapidesdans le cadre de l'e'valuationdiagnostique de l'autisme. Enfance 1, 133-142. IN: Gepner, B., \&Féron, F. (2009). Autism: a world changing too fast for a mis-wired brain? Neuroscience and biobehavioral reviews, 33(8), 1227-42. doi:10.1016/j.neubiorev.2009.06.006

Leekam, S. R., Nieto, C., Libby, S. J., Wing, L., \& Gould, J. (2007). Describing the sensory abnormalities of children and adults with autism. Journal of autism and developmental disorders, 37(5), 894-910. doi:10.1007/s10803-006-0218-7

Lepistö, T., Kuitunen, A., Sussman, E., Saalasti, S., Jansson-Verkasalo, E., Nieminenvon Wendt, T., \& Kujala, T. (2009).Auditory stream segregation in children with Asperger syndrome.Biological psychology, 82(3), 301-7. doi:10.1016/j.biopsycho.2009.09.004

Lepistö, T., Kujala, T., Vanhala, R., Alku, P., Huotilainen, M., \& Näätänen, R. (2005). The discrimination of and orienting to speech and non-speech sounds in children with autism. Brain research, 1066(1-2), 147-157. Elsevier. doi:10.1016/j.brainres.2005.10.052

Lepistö, T., Silokallio, S., Nieminen-von Wendt, T., Alku, P., Näätänen, R., \& Kujala, T. (2006). Auditory perception and attention as reflected by the brain eventrelated potentials in children with Asperger syndrome. Clinical neurophysiology: official journal of the International Federation of Clinical Neurophysiology, 117(10), 2161-71. doi:10.1016/j.clinph.2006.06.709

Lord, C., Rutter, M., DiLavore, P. C., \& Risi, S. (2001). Autism Diagnostic Observation Schedule - Generic. Los Angeles, CA: Western Psychological Services.

OramCardy, J. E., Flagg, E. J., Roberts, W., Brian, J., \& Roberts, T. P. L. (2005). Magnetoencephalography identifies rapid temporal processing deficit in autism and language impairment. Neuroreport, 16(4), 329.

Rosen, S. (1992). Temporal Information in Speech: Acoustic, Auditory and Linguistic Aspects. Philosophical Transactions of the Royal Society of London. Series B: Biological Sciences, 336 (1278), 367-373. doi:10.1098/rstb.1992.0070 
Stine, E. L., Wingfield, A., \& Poon, L. W. (1986). How much and how fast: rapid processing of spoken language in later adulthood. Psychology and aging, 1(4), 303-11.

Tardif, C., Thomas, K., Gepner, B., Rey, V., 2002. Contribution a' l'e'valuation du syste'mephonologiqueexplicite chez des enfantsautistes. Parole 21, 35-72. IN: Gepner, B., \&Féron, F. (2009). Autism: a world changing too fast for a miswired brain? Neuroscience and biobehavioral reviews, 33(8), 1227-42. doi:10.1016/j.neubiorev.2009.06.006

Tun, P. a. (1998). Fast noisy speech: age differences in processing rapid speech with background noise. Psychology and aging, 13(3), 424-34.

Tun, P. a, Wingfield, A., Stine, E. L., \&Mecsas, C. (1992). Rapid speech processing and divided attention: Processing rate versus processing resources as an explanation of age effects. Psychology and Aging, 7(4), 546-550. Elsevier.

Wallace, G. L., Dankner, N., Kenworthy, L., Giedd, J. N., \& Martin, A. (2010). Agerelated temporal and parietal cortical thinning in autism spectrum disorders.Brain : a journal of neurology, 133(Pt 12), 3745-54. doi:10.1093/brain/awq279

Wechsler, D. (1999). Wechsler Abbreviated Scale of Intelligence. San Antonio, TX: The Psychological Corporation.

Wechsler, D. (2008). Wechsler Adult Intelligence Scale-Fourth Edition. San Antonio, TX: Pearson.

Whitehouse, A.J., Bishop, D.V., 2008. Do children with autism 'switch off' to speech sounds? An investigation using event-related potentials. Developmental Science 11 (4), 516-524.

Wingfield, A. (1975). Acoustic redundancy and the perception of time-compressed speech. Journal of Speech \& Hearing Research, 18(1), 96-104.

Wingfield, A., Poon, L. W., Lombardi, L., \& Lowe, D. (1985). Speed of processing in normal aging: effects of speech rate, linguistic structure, and processing time. Journal of gerontology, 40(5), 579-85. 
Table 1. Participant Background Data Summary

\begin{tabular}{|c|c|c|c|c|c|}
\hline & \multicolumn{2}{|c|}{$\mathrm{ASD} N=19$} & \multicolumn{2}{|c|}{$\mathrm{TD} \mathrm{N}=19$} & \multirow[b]{2}{*}{$p$ values } \\
\hline & Mean (SD) & Range & Mean (SD) & Range & \\
\hline CA (months) & $482.79(136.00)$ & $285-716$ & $459.79(108.64)$ & $301-632$ & 0.568 \\
\hline \multicolumn{6}{|l|}{ Cognitive Correlates } \\
\hline WASI Full Scale & $113.37(15.27)$ & $78-133$ & $118.95(10.84)$ & $87-134$ & 0.203 \\
\hline WASI Verbal $^{\mathrm{a} 1}$ & $111.16(15.57)$ & $71-132$ & $115.58(11.52)$ & $83-135$ & 0.326 \\
\hline WASI Performance ${ }^{\mathrm{a} 2}$ & $112.95(12.97)$ & $92-129$ & $118.05(12.21)$ & $96-136$ & 0.221 \\
\hline $\mathrm{PPVT}^{\mathrm{b}}$ & $105.63(12.07)$ & $76-123$ & $106.05(10.24)$ & $84-125$ & 0.908 \\
\hline WM-Total ${ }^{\mathrm{c}}$ & $19.68(4.57)$ & $13-30$ & $19.16(4.69)$ & $13-28$ & 0.728 \\
\hline WM-Forward ${ }^{\mathrm{c} l}$ & $11.32(2.43)$ & $7-16$ & $11.53(2.32)$ & $8-15$ & 0.786 \\
\hline WM-Backward $^{\mathrm{c} 2}$ & $8.37(2.54)$ & $4-14$ & $7.63(2.98)$ & $4-13$ & 0.418 \\
\hline \multicolumn{6}{|l|}{ Behavioural Correlates } \\
\hline CC-SR-Total ${ }^{\mathrm{d}}$ & $67.84(33.28)$ & $32-159$ & $22.00(13.81)$ & $1-50$ & $<0.001^{*}$ \\
\hline CC-Lang. Struct. ${ }^{\mathrm{d} 1}$ & $14.58(12.19)$ & $1-49$ & $5.00(3.97)$ & $0-16$ & $<0.01 *$ \\
\hline CC-Pragmatics $\mathrm{d} 2$ & $17.84(11.35)$ & $0-39$ & $5.89(6.71)$ & $0-25$ & $<0.001^{*}$ \\
\hline CC-Social Eng. ${ }^{\mathrm{d}}$ & $35.42(12.58)$ & $19-71$ & $11.11(5.65)$ & $1-24$ & $<0.001^{*}$ \\
\hline Sensory Profile-Total ${ }^{\mathrm{e}}$ & $179.58(26.09)$ & $130-218$ & $131.89(28.36)$ & $32-160$ & $<0.001^{*}$ \\
\hline SP-Low Reg. ${ }^{\mathrm{el}}$ & $43.42(10.41)$ & $27-62$ & $26.16(6.23)$ & $10-35$ & $<0.001^{*}$ \\
\hline SP-Sensation Seek. ${ }^{\mathrm{e} 2}$ & $43.79(8.29)$ & $31-63$ & $47.58(9.88)$ & $12-58$ & 0.209 \\
\hline SP-Sensory Sens. ${ }^{\mathrm{e}}$ & $47.16(10.19)$ & $23-62$ & $29.05(8.18)$ & $4-39$ & $<0.001^{*}$ \\
\hline SP-Sensat. Avoid. ${ }^{\mathrm{e}}$ & $45.21(9.54)$ & $31-61$ & $29.11(9.31)$ & $6-48$ & $<0.001^{*}$ \\
\hline \multicolumn{6}{|l|}{ Clinical Correlates } \\
\hline AQ-Total ${ }^{\mathrm{i}}$ & $35.16(7.59)$ & $21-45$ & $12.26(5.45)$ & $3-21$ & $<0.001 *$ \\
\hline AQ-Social Skills ${ }^{\mathrm{f} 1}$ & $6.72(2.58)$ & $3-10$ & $1.32(1.38)$ & $0-4$ & $<0.001^{*}$ \\
\hline AQ-Atten. Switch ${ }^{\mathrm{f}}$ & $8.67(1.37)$ & $6-10$ & $3.26(1.79)$ & $0-6$ & $<0.001^{*}$ \\
\hline AQ-Atten. to Detail ${ }^{13}$ & $7.22(2.13)$ & $1-10$ & $3.58(2.10)$ & $0-7$ & $<0.001^{*}$ \\
\hline AQ-Commun. ${ }^{\mathrm{f}}$ & $6.50(2.55)$ & $2-10$ & $1.95(1.39)$ & $0-5$ & $<0.001 *$ \\
\hline AQ-Imagination ${ }^{\mathrm{f5}}$ & $6.22(2.29)$ & $2-10$ & $2.16(1.98)$ & $0-7$ & $<0.001 *$ \\
\hline ADOS-Diagnostic ${ }^{g}$ & $9.58(3.55)$ & $5-17$ & N/A & N/A & N/A \\
\hline ADOS-Commun. ${ }^{\mathrm{g} I}$ & $2.84(1.54)$ & $1-6$ & N/A & N/A & N/A \\
\hline ADOS-Soc. Int. ${ }^{\mathrm{g} 2}$ & $6.74(2.70)$ & $3-12$ & N/A & N/A & N/A \\
\hline ADOS-Imag..$^{3}$ & $1.05(0.70)$ & $0-2$ & N/A & N/A & N/A \\
\hline ADOS-Rep. Behav. ${ }^{\mathrm{g} 4}$ & $1.58(1.02)$ & $0-3$ & $\mathrm{~N} / \mathrm{A}$ & N/A & N/A \\
\hline
\end{tabular}

Note: $\mathrm{CA}=$ chronological age, $\mathrm{ASD}=$ autism spectrum disorders, $\mathrm{TD}=$ typically developing

${ }^{\mathrm{a}}$ Weschler Abbreviated Scales of Intelligence (WASI), standard score (Wechsler, 1999) ${ }^{\mathrm{a} 1}$ WASI Verbal IQ; ${ }^{\mathrm{a} 2}$ WASI Performance IQ

${ }^{\mathrm{b}}$ Peabody Picture Vocabulary Test (PPVT), standard score (Dunn \& Dunn, 1997)

${ }^{c}$ Working Memory Digit Span (WM), Weschler Adult Intelligence Scales, (Wechsler, 2008) ${ }^{\mathrm{c} 1}$ WM Forward Digit Span; ${ }^{\mathrm{c} 2} \mathrm{WM}$ Backward Digit Span

${ }^{\mathrm{d}}$ Communication Checklist - Self Report (CC-SR), raw score (Bishop et al., 2009) ${ }^{\mathrm{d} 1}$ CC-SR Language Structure; ${ }^{\mathrm{d} 2} \mathrm{CC}-\mathrm{SR}$ Pragmatics; ${ }^{\mathrm{d} 3} \mathrm{CC}-\mathrm{SR}$ Social Engagement

${ }^{\mathrm{e}}$ Adult/Adolescent Sensory Profile (SP), (Brown \& Dunn, 2002) ${ }^{\mathrm{e} 1}$ SP Low Registration; ${ }^{\mathrm{e} 2} \mathrm{SP}$ Sensation Seeking; ${ }^{\mathrm{e} 3} \mathrm{SP}$ Sensory Sensitivity; ${ }^{\mathrm{e} 4} \mathrm{SP}$ Sensation Avoiding

${ }^{\mathrm{f}}$ Adult Autism Spectrum Quotient (AQ), (Baron-Cohen et al., 2001) ${ }^{\mathrm{f1}} \mathrm{AQ}$ Social Skills; ${ }^{\mathrm{f} 2} \mathrm{AQ}$ Attention Switching; ${ }^{\mathrm{f3}} \mathrm{AQ}$ Attention to Detail; ${ }^{\mathrm{f4}} \mathrm{AQ}$ Communication; ${ }^{\mathrm{f}} \mathrm{AQ}$ Imagination

${ }^{\mathrm{g}}$ Autism Diagnostic Observation Schedule (ADOS), diagnostic total (Lord et al., 2001) ${ }^{\mathrm{g} 1} \mathrm{ADOS}$ Communication; ${ }^{\mathrm{g} 2} \mathrm{ADOS}$ Reciprocal Social Interaction; ${ }^{\mathrm{g} 3} \mathrm{ADOS}$ Imagination \& Creativity; ${ }^{g 4} \mathrm{ADOS}$ Repetitive Behaviours 
Table 2. Mean percentage correct and discrepancv scores, standard deviations, \& ranges

\begin{tabular}{|c|c|c|c|c|c|}
\hline & \multicolumn{2}{|c|}{$\mathrm{ASD}$} & \multicolumn{2}{|c|}{ TD } \\
\hline & & Mean (SD) & Range & Mean (SD) & Range \\
\hline Accuracy & Normal & $81.72(15.77)$ & $40.67-98.67$ & $80.38(12.26)$ & $51.33-95.33$ \\
\hline Percentage & Moderate & $80.63(13.86)$ & $50.00-97.33$ & $82.42(12.62)$ & $40.67-93.33$ \\
\hline Correct & Fast & $77.16(17.32)$ & $34.67-96.00$ & $78.07(12.06)$ & $50.67-90.67$ \\
\hline Scores & Total & $79.84(14.94)$ & $44.22-97.33$ & $80.29(11.45)$ & 48.89-92.44 \\
\hline Accuracy & Moderate & $1.09(9.12)$ & $-16.67-18.00$ & $-2.03(7.23)$ & $-18.67-10.67$ \\
\hline $\begin{array}{c}\text { Discrepancy } \\
\text { Scores } \\
\end{array}$ & Fast & $2.56(8.14)$ & $-12.67-23.33$ & $2.31(8.56)$ & $-12.00-26.67$ \\
\hline Recall & Normal & $77.35(21.48)$ & $51.50-142.70$ & $75.52(68.60)$ & $47.10-111.10$ \\
\hline Time & Moderate & $76.00(25.30)$ & $48.20-152.00$ & $68.60(10.55)$ & $53.90-91.90$ \\
\hline Scores & Fast & $68.46(21.41)$ & $45.00-130.60$ & $71.43(10.87)$ & $56.80-91.90$ \\
\hline & Total & $221.81(62.26)$ & $151.40-353.40$ & $215.55(32.23)$ & $166.00-274.80$ \\
\hline $\mathrm{RT}$ & Moderate & $1.34(17.92)$ & $-54.50-35.50$ & $6.92(13.86)$ & $-20.70-36.70$ \\
\hline $\begin{array}{c}\text { Discrepancy } \\
\text { Scores }\end{array}$ & Fast & 8.89 (14.59) & $-23.30-50.70$ & $4.09(10.64)$ & $-14.40-21.80$ \\
\hline
\end{tabular}

Table 4.Summary of significant correlations between fast speed discrepancy scores and background measures

\begin{tabular}{|c|c|c|}
\hline & ASD & TD \\
\hline \multicolumn{3}{|l|}{ Cognitive Correlates } \\
\hline PIQ & $-0.513 *$ & -0.030 \\
\hline \multicolumn{3}{|c|}{ Behavioural Correlates } \\
\hline \multicolumn{3}{|c|}{ Communication Checklist } \\
\hline Language Structure & $0.654 * *$ & 0.197 \\
\hline Pragmatic Skills & $0.481 *$ & 0.273 \\
\hline Social Engagement & $0.717 * *$ & 0.132 \\
\hline Total Score & $0.675 * *$ & 0.243 \\
\hline \multicolumn{3}{|l|}{ Sensory Profile } \\
\hline Low Registration & $0.654 * *$ & 0.125 \\
\hline Sensory & $0.538 *$ & 0.385 \\
\hline Total Score & $0.544 *$ & 0.248 \\
\hline \multicolumn{3}{|l|}{ Clinical Correlates } \\
\hline AQ & & \\
\hline Social Skills & $0.480 *$ & -0.150 \\
\hline Imagination & $0.580 *$ & -0.007 \\
\hline Chronological Age & $0.739 * * *$ & -0.149 \\
\hline
\end{tabular}

Note: ${ }^{*} p<0.05,{ }^{* *} p<0.01,{ }^{* * *} p<0.001$ (two-tailed)

Negative correlations indicate a relationship between higher scores on the background measure and reduced perceptual disturbance from speed 
Table 4. Multiple regression statistics for predictor variables to accuracy discrepancy score of ASD participants during fast speed condition

\begin{tabular}{lccccc}
\hline & $B$ & SE $B$ & $\beta$ & $t$ & $p$ \\
\hline Age & 0.04 & 0.01 & 0.62 & 7.09 & $0.000^{* * *}$ \\
CC-Social Engagement & 0.19 & 0.09 & 0.28 & 2.13 & 0.053 \\
SP-Sensory Sensitivity & 0.40 & 0.12 & 0.47 & 3.23 & $0.007^{*} *$ \\
AQ- Social Skills & -0.70 & 0.38 & -0.22 & -0.18 & 0.092 \\
\hline
\end{tabular}

Note: $\mathrm{B}=$ un-standardised beta coefficient, $\mathrm{SE} \mathrm{B}=$ standard error, $\beta=$ standardised beta coefficient, $\mathrm{t}=\mathrm{t}$-test statistic, $p=$ significance value

\section{FIGURES}



Figure 1.Group x speed manipulation interaction

Note: Negative recall times indicate increased perceptual disturbance from speed 